\title{
Clinical REsearch During Outbreaks (CREDO) Training for Low- and Middle-Income Countries
}

\author{
Nzelle Delphine Kayem, Amanda Rojek, \\ Emmanuelle Denis, Alex Salam, \\ Andreas Reis, Piero Olliaro, Peter Horby
}

We describe a pilot of the Clinical REsearch During Outbreaks (CREDO) initiative, a training curriculum for researchers in epidemic-prone low- and middle-income countries who may respond to disease outbreaks. Participants reported improved confidence in their ability to conduct such research and overall satisfaction with the course structure, content, and training.

C Mlinical research is an essential component of outbreak response because it underpins clinical management and public health measures (1). However, conducting clinical research during epidemics is challenging $(2,3)$. The Ebola epidemic in West Africa underscored the need for improved research infrastructure and capacity in epidemic-prone vulnerable low- and middle-income countries (LMICs) (4). To address this need, a training curriculum, Clinical REsearch During Outbreaks (CREDO), was jointly developed by the Special Programme for Research and Training in Tropical Diseases (TDR), hosted by the World Health Organization, the International Severe Acute Respiratory and Emerging Infections Consortium, and the United Kingdom Public Health Rapid Support Team. The main objective of the CREDO training curriculum is to strengthen the national capacities of LMICs to design and implement clinical research during outbreaks of infectious diseases and to improve team capacity and team effort. We describe the development and piloting of the CREDO training curriculum and present the results of the formal evaluation.

\section{The Study}

The learning objectives for the CREDO curriculum (Table 1) were developed using the TDR Global Competency Framework for Clinical Research $(5,6)$, which describes all areas of competency required to conduct clinical research (6). The content of the modules addressed both observational

Author affiliations: University of Oxford, Oxford, UK (N.D. Kayem, A. Rojek, E. Denis, A. Salam, P. Olliaro, P. Horby); United Kingdom Public Health Rapid Support Team, Oxford (A. Salam); World Health Organization, Geneva, Switzerland (A. Reis) studies and clinical trials. Observational studies were included because they are essential for understanding the etiology, natural history, and pathophysiology of epidemic infectious diseases, many of which are poorly understood. Clinical trials address questions on therapeutics, diagnostics, and other topics, and CREDO covers a range of trial designs including adaptive designs, which may be better suited to the evolving nature of outbreaks.

CREDO is structured as a blended-learning format, with face-to-face sessions (workshops) and asynchronous, downloadable online sessions (e-learning). Our training model was designed with the workshops occurring before and after the e-learning component to introduce and then solidify knowledge gained in the online component. Our assessment model combined multiple-choice questions in the e-learning sessions with simulation exercises in the workshop sessions to provide a balance in testing between recall and critical or creative thinking (7).

The curriculum has 12 modules (Table 2): 1 workshop and 11 e-learning modules, 2 of which are prerequisites that must be completed before the workshop date. Modules are freely available online through a digital platform suited to the low bandwidths found in LMICs. The e-learning component is hosted on the Global Health Network website (https://isaric.tghn.org/credo). It is self-paced over a period of 5-6 months, and each module takes an average of 1-2 hours, with some taking as many as $4-5$ hours, to complete. The e-learning is completed individually; participants complete the final assessment during the second workshop as a team, each team member actively using the knowledge learned from the online modules to contribute to the design of a clinical trial during a hypothetical outbreak. Each participant who successfully completes all course components earns a CREDO certificate.

We designed the curriculum for team training with multidisciplinary and intact teams (8); that is, teams that are already working together to develop and implement clinical studies. Team training refers to the training of an entire team and has resulted in improved patient outcomes in various areas of medicine, such as trauma $(8,9)$.

During March-August 2017, we conducted a pilot of the CREDO training curriculum. An informal call invited team applications from sub-Saharan Africa, targeted because populations in the region are at risk for outbreaks from high-threat infectious diseases (10). We selected 19 
Table 1. Learning objectives for the Clinical REsearch During Outbreaks (CREDO) training course

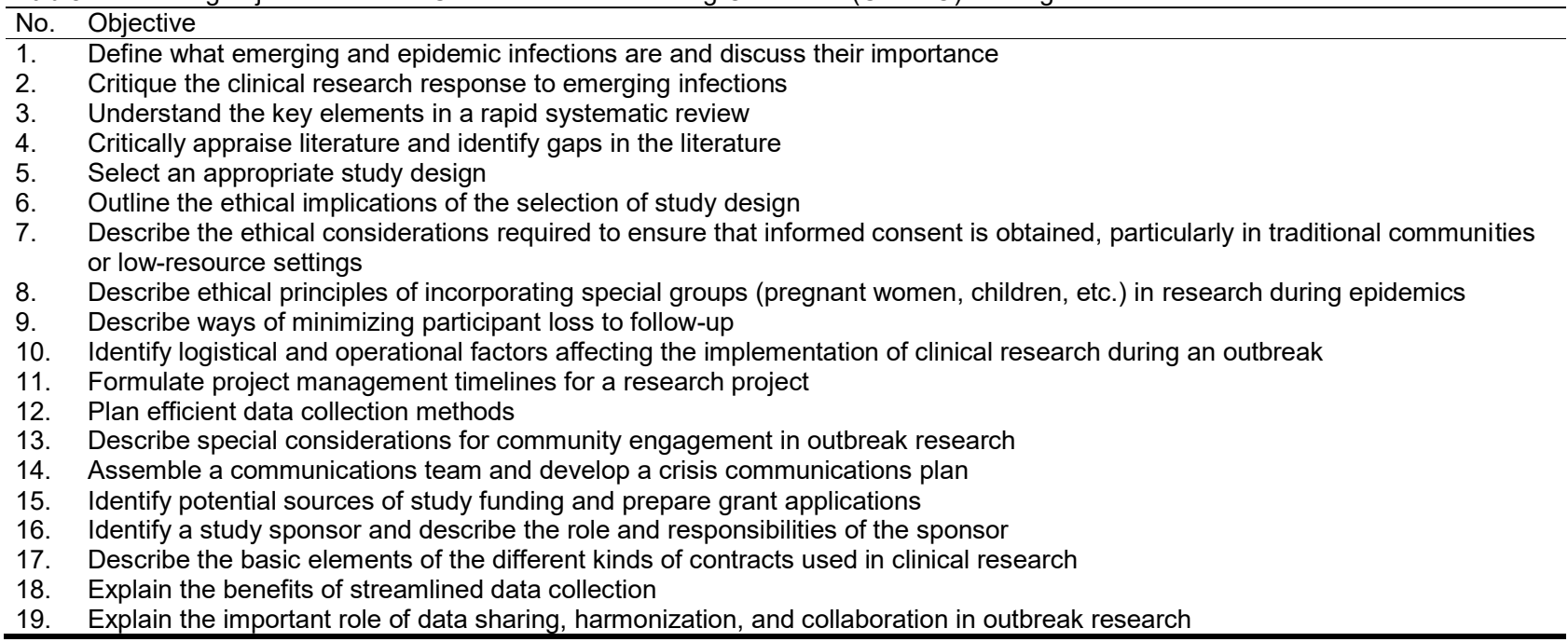

Table 2. Curriculum structure of Clinical REsearch During Outbreaks (CREDO) training course*

Module title Module summary and website link

Prerequisite modules

Good clinical practice

The global health research process map

Workshop: Evidence-based medicine for epidemic infections and key issues in study design

E-learning component: self-paced, in any order, comp Rapid evidence-needs appraisal

Research study planning and governance

Study design

Statistics

Logistics and operational planning

Data sharing and harmonization

Ethics

Communications and engagement

Special groups: Children, pregnant women, mother/child
A framework of principles to ensure the safety of research participants and integrity and validity of data: https://globalhealthtrainingcentre.tghn.org/ich-good-clinicalpractice

A pragmatic interactive tool provides step-by-step guidance for each stage that needs to be considered when planning a new study: https://processmap.tghn.org/about

Delivered in 3 presentations, it provides an introduction to epidemic and emerging infections, critiques the clinical research responses to previous outbreaks, highlights the challenges to clinical research during outbreaks, and discusses possible mitigating strategies: https://isaric.tghn.org/credo/credo-workshop-1

A guide for the conduct of rapid reviews in the event of an outbreak:

https://globalhealthtrainingcentre.tghn.org/credo-rapid-evidence-needs-appraisal

An overview of how to set up a clinical research study and find and apply for funding, and important concepts for study managements:

https://globalhealthtrainingcentre.tghn.org/credo-research-study-planning-andgovernance

An introduction to the challenges to research design during outbreaks and some mitigating strategies: https://globalhealthtrainingcentre.tghn.org/credo-study-design

Background of statistical principles relevant to clinical research and trial design and challenges in outbreaks and some solutions:

https://globalhealthtrainingcentre.tghn.org/credo-statistics

Pragmatic solutions to common logistical and operational challenges to research in outbreaks: https://globalhealthtrainingcentre.tghn.org/credo-logistical-andoperational-planning

General outline of data management, sharing, and harmonization to guide the conduct of research during outbreaks: https://isaric.tghn.org/credo/credo-datasharing-and-harmonisation

WHO course on ethics in outbreaks; modules 2,4 , and 6 on the TGHN platform: https://globalhealthtrainingcentre.tghn.org/research-ethics-epidemics-pandemicsand-disaster-situations

Effective communication and engagement during outbreaks:

https://globalhealthtrainingcentre.tghn.org/credo-communications-and-communityengagement

A consensus statement on the inclusion of children and pregnant women in research in disease outbreaks to help facilitate including these important groups in future research and clinical trials: https://isaric.tghn.org/credo/ethics-special-groups 
Figure 1. Self-assessed level of confidence with learning objectives of Clinical REsearch During Outbreaks (CREDO) before and after course. Participants' level of confidence in their ability to implement a clinical research study during an outbreak changed substantially: in the precourse assessment, 3 of 17 participants rated themselves as confident or very confident; in postcourse assessment, 16 of 17 did.

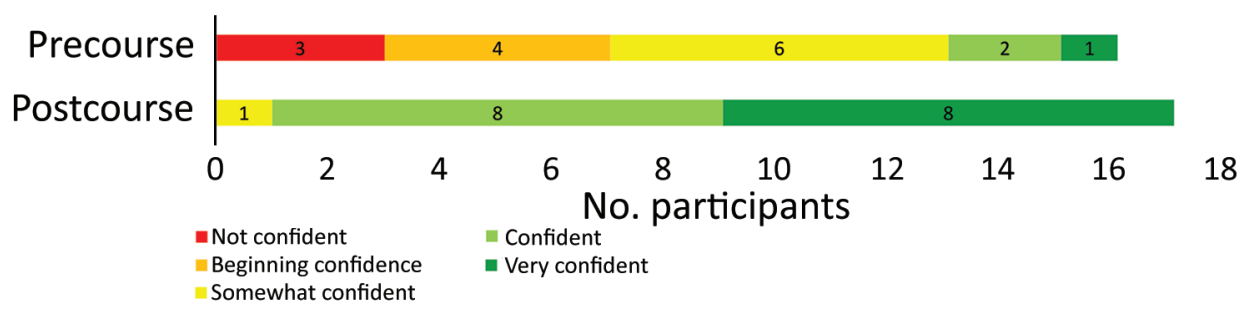

participants from 4 multidisciplinary teams in 4 countries (Ethiopia, Ghana, Côte d'Ivoire, and Uganda) on the basis of their clinical research experience and team diversity. Of the selected participants, 8 were female and 11 male. Teams consisted of 11 medical doctors, 2 research nurses, 2 data managers, 1 clinical trial manager, and 3 biomedical scientists.

We conducted the first workshop in Entebbe, Uganda. Its purpose was to appraise the challenges of outbreak research; describe the fundamental concepts of generating clinical evidence; introduce the online component of the training; and assess, using simulated team-based exercises, the participants' confidence in planning and conducting clinical research during an outbreak. After the workshop, participants had time to complete the e-learning modules.

The closing workshop was conducted in Addis Ababa, Ethiopia. Two weeks before this workshop, we gave participants an assignment to develop a clinical trial protocol for a hypothetical outbreak (available on the course website, https://isaric.tghn.org/credo/credo-overall-assignment). This workshop increased and assessed the participants' knowledge and understanding of observational research and clinical trials. The clinical trial protocols in the assignment were assessed using role play; facilitators played the roles of statisticians, community representatives, scientific experts, ethical reviewers, and government representatives. The teams then incorporated suggestions made by the faculty. The revised protocols then underwent review by peers using an adapted scoring tool (Appendix, https://wwwnc. cdc.gov/EID/article/25/11/18-0628-App1.pdf).

Pilot participants completed an online precourse and postcourse evaluation form anonymously. The questions were based on the learning objectives (Table 1) and sought the participants' views on the training received, the structure and the content of the individual course modules, and their ability to conduct clinical research during an outbreak. The responses were structured on a Likert scale from strongly agree to strongly disagree or not confident to very confident, with free-text space to clarify any responses (Appendix).

Of the 19 participants, $16(84.2 \%)$ completed the precourse questionnaire and $17(89.5 \%)$ completed the postcourse questionnaire. Most participants were satisfied with the course; 16 participants rated their level of satisfaction as satisfied or very satisfied. Self-assessed levels of confidence increased after the course; 16 participants rated their level of confidence in implementing the course objectives as confident or very confident after the course, compared with only 3 participants before the course (Figure 1). However, another evaluation is required during or after an outbreak to better assess the effectiveness of the course.

An assessment of the content of CREDO showed largely positive reviews, particularly with respect to the relevance and adequacy of the material covered in each of the course modules (Figure 2). Time for completion of each module was generally sufficient except in the rapid evidence appraisal module, for which 3 participants indicated time was inadequate. Some of the major strengths of CREDO were its structure and content, which most participants considered "holistic" and "comprehensive."

\section{Conclusions}

Although clinical research is becoming recognized as an important component of outbreak management $(1,11)$, few resources exist to assess or build capacity in this area. Most

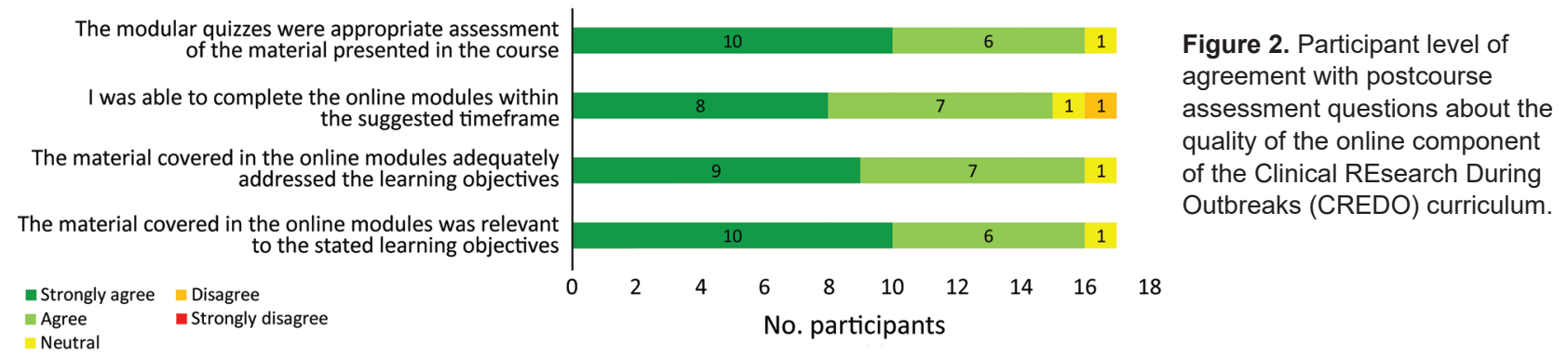


of the available outbreak training programs or courses focus on surveillance, epidemiology, outbreak investigation, or laboratory investigation (12-15). CREDO offers a unique perspective with its focus on clinical research and clinical trials.

The strengths of the CREDO training curriculum lie in its format of blended learning, team-based approach, and content tailored to outbreak conditions. Its combination of training in observational studies and clinical trials builds capacity for collecting the much-needed information on pathophysiology and natural history of poorly known conditions, essential components to identify the right interventions to test and the design of the intervention trials.

In addition, CREDO, as an open-access resource, lends itself to adoption and adaptation by interested sites and to creating a community of science by sharing additional or modified materials through the Global Health Network website. CREDO resources can be incorporated into medical or public health training curricula or used as a standalone course for continuous professional development. Some ways in which CREDO will become sustainable are by broadening the number of trainers from among course participants, supporting country ownership, and franchising the course through a variety of providers.

\section{Acknowledgments}

We thank the Global Health Network for the use of and assistance with their online platform; the entire CREDO faculty (https://isaric.tghn.org/credo) for developing the online modules and workshop exercises; the participants from the 4 country teams who took part in the pilot course; and the CREDO core development team (Katherine Littler, Nikki Shindo, Michael Mawanda, Bin Cao, Fernando Bozza, Proochista Ariana, and Daniel Bausch) who advised on the development of the curriculum.

Funding for this work was provided by the UNICEF/UNDP/ World Bank/WHO Special Programme for Research and Training in Tropical Diseases and the UK Public Health Rapid Support Team (funded by the government of the United Kingdom). The funders had no role in the decision to publish or preparation of the manuscript. N.D.K. is a Commonwealth Scholar funded by the UK Department for International Development. P.O. is a previous staff member of the World Health Organization and the UNICEF/UNDP/World Bank/WHO Special Programme for Research and Training in Tropical Diseases.

\section{About the Author}

Dr. Kayem is a physician and a UK Commonwealth Scholar at the University of Oxford studying for a DPhil in clinical medicine. Her research interests are emerging infectious diseases and their effects on vulnerable populations, and improving the response to disease outbreaks in Africa.

\section{References}

1. Lurie N, Manolio T, Patterson AP, Collins F, Frieden T. Research as a part of public health emergency response. N Engl J Med. 2013;368:1251-5. https://doi.org/10.1056/NEJMsb1209510

2. Rojek AM, Horby PW. Modernising epidemic science: enabling patient-centred research during epidemics. BMC Med. 2016;14:212. https://doi.org/10.1186/s12916-016-0760-x

3. Rojek AM, Horby PW. Offering patients more: how the West Africa Ebola outbreak can shape innovation in therapeutic research for emerging and epidemic infections. Philos Trans R Soc Lond B Biol Sci. 2017;372:20160294. https://doi.org/10.1098/rstb.2016.0294

4. Koita OA, Murphy RL, Fongoro S, Diallo B, Doumbia SO, Traoré $\mathrm{M}$, et al. Clinical research and the training of host country investigators: essential health priorities for disease-endemic regions. Am J Trop Med Hyg. 2016;94:253-7. https://doi.org/ 10.4269/ajtmh.15-0366

5. Julé A, Furtado T, Boggs L, van Loggerenberg F, Ewing V, Vahedi M, et al. Developing a globally applicable evidenceinformed competency framework to support capacity strengthening in clinical research. BMJ Glob Health. 2017;2:e000229. https://doi.org/10.1136/bmjgh-2016-000229

6. World Health Organization Special Programme for Research and Training in Tropical Diseases. Using the TDR global competency framework for clinical research: a set of tools to help develop clinical researchers. 2016 [cited 2019 Aug 19]. https://www.who. int/tdr/publications/year/2016/competency-framework-clinical-res

7. Hift RJ. Should essays and other "open-ended"-type questions retain a place in written summative assessment in clinical medicine? BMC Med Educ. 2014;14:249. https://doi.org/10.1186/ s12909-014-0249-2

8. Salas E, DiazGranados D, Klein C, Burke CS, Stagl KC, Goodwin GF, et al. Does team training improve team performance? A meta-analysis. Hum Factors. 2008;50:903-33. https://doi.org/ 10.1518/001872008X375009

9. Capella J, Smith S, Philp A, Putnam T, Gilbert C, Fry W, et al. Teamwork training improves the clinical care of trauma patients. J Surg Educ. 2010;67:439-43. https://doi.org/10.1016/ j.jsurg.2010.06.006

10. Pigott DM, Deshpande A, Letourneau I, Morozoff C, Reiner RC Jr, Kraemer MUG, et al. Local, national, and regional viral hemorrhagic fever pandemic potential in Africa: a multistage analysis. Lancet. 2017;390:2662-72. https://doi.org/10.1016/S0140-6736(17)32092-5

11. National Academies of Sciences, Engineering, and Medicine. Integrating clinical research into epidemic response: the Ebola experience. Washington, DC: The National Academies Press; 2017. https://doi.org/10.17226/24739.

12. Public Health England. United Kingdom Field Epidemiology Training Programme prospectus. 2019 [cited 2019 July 8]. https://www.gov.uk/ guidance/field-epidemiology-training-programme-fetp

13. Lescano AG, Salmon-Mulanovich G, Pedroni E, Blazes DL. Outbreak investigation and response training. Science. 2007;318:574-5. https://doi.org/10.1126/science.1146837

14. Centers for Disease Control and Prevention. Field Epidemiology Training Program (FETP) training modules and resources. 2019 [cited 2019 July 8]. https://www.cdc.gov/globalhealth/ healthprotection/fetp/training_modules/index.html

15. Centers for Disease Control and Prevention; Society for Healthcare Epidemiology of America. CDC/SHEA Outbreak Response Training Program (ORTP). 2019 [cited 2019 July 8]. http://ortp.shea-online.org/

Address for correspondence: Nzelle Delphine Kayem, University of Oxford-Centre for Tropical Medicine and Global Health, Wellcome Centre for Human Genetics, Roosevelt Dr, Oxford OX3 7BN, UK; email: nzelle.kayem@ndm.ox.ac.uk 